\title{
General Discussion to Papers of Prof Sussman and Dr Young et al.
}

DR H. FRANKEL (G.B.). It's very flattering of John to use our classifications for comparative purposes, and the results I agree show really no significant difference in view of the fact that many different people performed the neurological examinations and that our study was a retrospective one. The reason we chose these five grades was that we felt it was the maximum that one could pull out of a significant number of our notes retrospectively and also it was the most data that we could handle manually and we weren't trained to do anything other than handle data on sheets of paper writing down numbers in bits. In order to answer the questions, the interesting questions raised by John we in fact have to do joint prospective studies and the data must be handled by a more sophisticated system.

Sir Ludwig GutTManN (G.B.). First to John's interesting paper. I would like to ask if he could give us more details about the surgical procedures and the effect of the surgery immediately on the general condition, particularly on the autonomic mechanisms -intestinal, bladder and respiration. If you compare your statistics with my own which I gave in 1963 in Edinburgh you might find even a closer agreement, because in that statistic I was concerned with patients who were admitted within the first 24 or 48 hours.

With regard to that very important paper of Professor Sussman, a very experienced neurosurgeon, I only can say from my own observations I agree with every word he said. When people arrive in the emergency room, they are examined not infrequently by inexperienced residents who do not ask immediately for a detailed neurological examination. That is an unsatisfactory procedure in the initial management of the acute spinal cord damaged patient. The other point is the way the first X-ray is taken of the spine. Patients are sent to the X-ray department without the doctor in charge of the case being himself in attendance. In cervical injuries the head is often immobilised merely by sandbags even if the patient is semi-conscious and restless and moves his head not only to the sides but also anteriorly. With regard to the X-ray itself, we are so indoctrinated to look at the lateral X-ray that we don't consider carefully enough the a.p. I have seen, as no doubt many of you have, that the lateral X-ray may reveal what appears to be a simple compression fracture with or without a little shearing off of the anterior part of the vertebral body, yet the a.p. will show, if you look carefully, some damage of the articulations, which immediately exposes the real severity of the fracture dislocation which, if not treated immediately by skull traction or at least Glisson halter, may become unstable. If that patient is not immobilised properly by head traction then of course all the drama of transforming an incomplete into a complete lesion will follow.

Another point I'd like to mention is the form of consent by the patient and, in particular, relatives, for surgical procedures. The patient is very often in a shocked state and may be unconscious or semi-conscious. I feel it is the responsibility of the surgeon to tell the relatives in some detail what he is going to do including the risks of the immediate operation and, moreover, the reason of emergency and whether there are alternatives, so that the relatives may decide whether the surgeon should postpone the operation or whether they wish an alternative procedure first. This is, I feel, important from a legal point of view, especially in the United States where the lawyers not infrequently make an issue of insufficient information, which may place the surgeon in an awkward situation in the courts. Most of you know that it was the American Association of Neurological Surgeons who in 1975 published a document in which legal problems and their consequences were discussed. The compensation for malpractice is enormous. Alas-the tragedy is that only 15-25 per cent goes to the patient, and the rest goes to the lawyers and the court. That is why Sussman's paper is important and very timely. 